\title{
Investigation on extreme frequency shift in silica fiber-based high-power Raman fiber laser
}

\author{
Jiaxin Song, Hanshuo Wu, Jun Ye, Hanwei Zhang, Jiangming Xu, Pu Zhou, and Zejin Liu \\ College of Optoelectronic Science and Engineering, National University of Defense Technology, Changsha 410073, China \\ (Received 29 January 2018; revised 9 March 2018; accepted 19 March 2018)
}

\begin{abstract}
In this paper, we experimentally investigated the extreme frequency shift in high-power Raman fiber laser (RFL). The RFL was developed by using a pair of fiber Bragg gratings with fixed and matched central wavelength (1120 nm) combined with a piece of 31-m-long polarization maintaining (PM) passive fiber adopted as Raman gain medium. The pump source was a homemade high-power, linearly polarized (LP) wavelength-tunable master oscillator power amplifier (MOPA) source with $25 \mathrm{~nm}$ tunable working range (1055-1080 nm). High-power and high-efficiency RFL with extreme frequency shift between the pump and Stokes light was explored. It is found that frequency shift located within $10.6 \mathrm{THz}$ and $15.2 \mathrm{THz}$ can ensure efficient Raman lasing, where the conversion efficiency is more than $95 \%$ of the maximal value, $71.3 \%$. In addition, a maximum output power of $147.1 \mathrm{~W}$ was obtained with an optical efficiency of $71.3 \%$, which is the highest power ever reported in LP RFLs to the best of our knowledge.
\end{abstract}

Keywords: linearly polarized laser; Raman fiber laser; Raman gain spectrum

\section{Introduction}

Raman fiber laser (RFL) can theoretically achieve emission at almost arbitrary wavelength with the help of proper pump wavelength ${ }^{[1]}$; thus, the emission range of RFL is much broader than lasers based on rare earth-doped fibers. Therefore, RFL has been explored to achieve high-power output at specialized wavelengths ${ }^{[2-10]}$, which are rather challenging for lasing or amplifying efficiently by rare earthdoped fibers ${ }^{[11-13]}$. To date, high-power RFLs have been widely investigated and applied in optical pumping, frequency conversion, optical communications, biology and scientific research ${ }^{[14-22]}$. It is well known that the Raman gain in silica fibers extends over a large frequency range up to $40 \mathrm{THz}^{[23]}$. In general, the frequency shift between pump wavelength and Raman wavelength is approximately 13.2 or $14.7 \mathrm{THz}$ corresponding to the double-peak structure of Raman gain spectrum for silica fiber ${ }^{[23,24]}$. The frequency shift between the pump light and target Raman light is usually designed to be well matched to fulfill the peaks of Raman gain spectrum. For example, hundred-watt-level high-power RFLs operating at $1120 \mathrm{~nm}$ and $1150 \mathrm{~nm}$ were demonstrated by using $1070 \mathrm{~nm}$ and $1090 \mathrm{~nm}$ fiber lasers as pump sources, respectively ${ }^{[3,17]}$. It is to be noted that, for ap-

Correspondence to: P. Zhou, College of Optoelectronic Science and Engineering, National University of Defense Technology, No. 109 Deya Road, Changsha 410073, China. Email: zhoupu203@163.com plication that requires RFLs with some specific wavelengths, it is usually not easy to obtain high-power pump source. The central wavelength of the pump source should match well with a $13.2 \mathrm{THz}$ frequency shift compared with the output wavelength of RFL. For example, single mode $1178 \mathrm{~nm}$ RFL, which can be used for frequency doubling to the yellow, is often pumped by $1120 \mathrm{~nm}$ fiber laser. However, it is relatively difficult to achieve high-power lasing at $1120 \mathrm{~nm}$ based on Yb-doped fiber because of smaller net gain ${ }^{\text {[25-27] }}$ compared with shorter wavelength ${ }^{[28,29]}$. Therefore, it is interesting to explore the feasibility of generating highpower $1178 \mathrm{~nm}$ by pumping with a powerful Yb-doped fiber laser (YDFL) operating at $\sim 1110 \mathrm{~nm}$ (or even shorter wavelength) corresponding to the frequency shift of $\sim 15.6 \mathrm{THz}$, which has a significant difference compared with $13.2 \mathrm{THz}$. In fact, efficient lasing from a fiber Raman oscillator by fully exploring the broadband gain spectrum was studied as early as in $1977^{[30,31]}$. In the visible band, tunable Raman oscillator pumped by a $4 \mathrm{~W}$ argon ion laser at $514.5 \mathrm{~nm}$ was tuned over $8 \mathrm{~nm}$ using a prism, which corresponds to the frequency shift from 6.2 to $14.9 \mathrm{THz}$. In the infrared band, Stokes oscillation tuning from 1085 to $1130 \mathrm{~nm}$ corresponding to the frequency shift of 5.5 to $16.5 \mathrm{THz}$ was obtained pumped by a $5 \mathrm{~W}$ Nd:YAG laser at $1064 \mathrm{~nm}$. In 2008, Belanger et al. demonstrated a widely tunable RFL, the frequency shift of which ranged from 2.9 to $17.6 \mathrm{THz}^{[32]}$. Almost constant output Stokes power, up to $5.0 \mathrm{~W}$, was obtained 


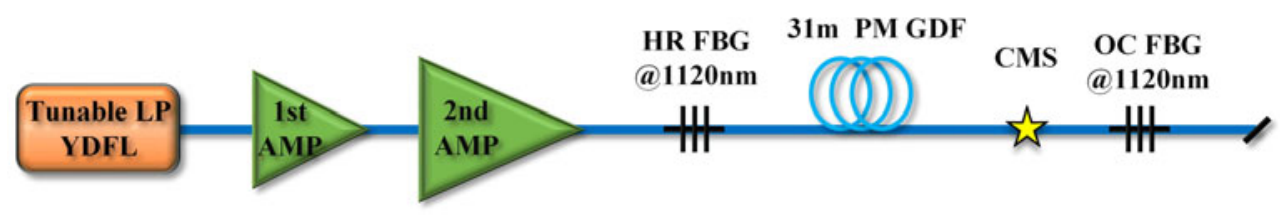

Figure 1. The experimental schematic of the linearly polarized Raman fiber laser. LP: linearly polarized; AMP: amplifier; GDF: germanium-doped fiber; CMS: cladding mode stripper.

when the frequency shift was from 10.5 to $14.0 \mathrm{THz}$. The output power of reported results is relatively low. However, high-power tunable RFL is required in some applications, such as nonlinear frequency conversion. In addition, the broad Raman gain spectral profile at high-power level needs to be further explored. When the frequency shift deviates significantly from the peak value, whether high-power and high-efficiency RFL could be achieved has not been studied in detail to date, to the best of our knowledge.

In this paper, efficient high-power Raman lasing with extreme frequency shift between the pump and Stokes light was explored experimentally, based on an RFL cavity with fixed central wavelength. A homemade highpower wavelength-tunable master oscillator power amplifier (MOPA) with $\sim 25 \mathrm{~nm}$ tunable working range was employed as pump source. All the fiber and fiber components involved are polarization maintained to eliminate the influence of polarization state. It is found that frequency shift located within $10.6 \mathrm{THz}$ and $15.2 \mathrm{THz}$ can ensure efficient Raman lasing, where the conversion efficiency is more than $95 \%$ of the maximal value of $71.3 \%$. In addition, a maximum output power of $147 \mathrm{~W}$ was obtained with an optical efficiency of $71.3 \%$ at the pump wavelength of $1062.5 \mathrm{~nm}$. The polarization extinction ratio (PER) of RFL is around $20 \mathrm{~dB}$. This is the highest power ever reported in LP Raman fiber oscillators to the best of our knowledge. Before this, Surin et al. reported $65 \mathrm{~W}$ output power in linearly polarized (LP) RFL operating at $1178 \mathrm{~nm}$, which is the highest-power in LP Raman fiber oscillators so far, as far as we know ${ }^{[21]}$. It is to be noted that the Raman Laser Module (RLM) and Raman Laser Rack (RLR) series produced by IRE Polus Group (IPG) Photonics Corporation can deliver up to $500 \mathrm{~W}$ RFL with optional LP operation ${ }^{[33]}$. However, the detailed configuration has not been revealed. In this case, the comparison is not taken into consideration.

\section{Experimental setup}

The extreme frequency shift between the pump wavelength and Raman wavelength with high efficiency in high-power RFL was investigated in the experiment. As is shown in Figure 1 , the experimental setup of the $\sim 150 \mathrm{~W}$ level LP RFL was designed. The classical oscillator configuration was adopted because it has been well studied and it is beneficial to exclude the effect of other factors. A pair of fiber Bragg gratings (FBGs) centering at $1120 \mathrm{~nm}$ were adopted to fix the wavelength of the target Raman laser. The reflectivity of the high reflectivity (HR) FBG and the output coupler (OC) FBG is $99.6 \%$ and $50 \%$ at $1120 \mathrm{~nm}$, with the full width at half maximum (FWHM) bandwidth of $1.5 \mathrm{~nm}$ and $0.45 \mathrm{~nm}$, respectively. The FBGs were written on polarization maintaining (PM) fiber with $10 \mu \mathrm{m} / 125 \mu \mathrm{m}$ core/cladding diameter. A piece of commercial 31-m-long PM passive fiber (germanium-doped fiber, GDF) was selected to provide Raman gain, the core and inner cladding diameters of which are 10 and $125 \mu \mathrm{m}$, respectively. The pump source is a homemade tunable 200-watt-level MOPA source with LP laser output, which is seeded by an LP YDFL and boosted by two stage amplifiers. LP configuration was designed because Raman gain is polarization-dependent, so in the experiment we can investigate the spectrum dependence of Raman gain regardless of the influence of polarization state. Cladding mode stripper (CMS) was achieved by coating high refractive index gel around the splicing point between the GDF and OC FBG to strip the cladding light and consequently protects the OC FBG. The output end was angle-cleaved to $8^{\circ}$ to suppress unexpected backward reflection.

The tuning range of the pump source was from $1055 \mathrm{~nm}$ to $1080 \mathrm{~nm}$. $1067.4 \mathrm{~nm}$ laser wavelength would be preferred because of the well-matched frequency shift $(13.2 \mathrm{THz})$ compared with $1120 \mathrm{~nm}$. Nevertheless, deviating from $1067.4 \mathrm{~nm}$ provides a flexible platform to investigate the gain property of RFL. The output properties of the tunable pump source were measured and recorded. As is shown in Figure 2(a), the pump source has $25 \mathrm{~nm}$ tuning range (from 1055 to $1080 \mathrm{~nm}$ ), the FWHM linewidth of which, at different wavelengths, are all around $0.6 \mathrm{~nm}$. Figure 2(b) depicts the maximum power of the pump source as a function of wavelength, which is all around $200 \mathrm{~W}$. By recording and analyzing the output properties, the extreme frequency shift and the operating state of the Raman laser at different pump wavelengths were investigated.

\section{Results and discussion}

By comparing the output power and the corresponding conversion efficiency at different pump wavelengths, the 
(a)

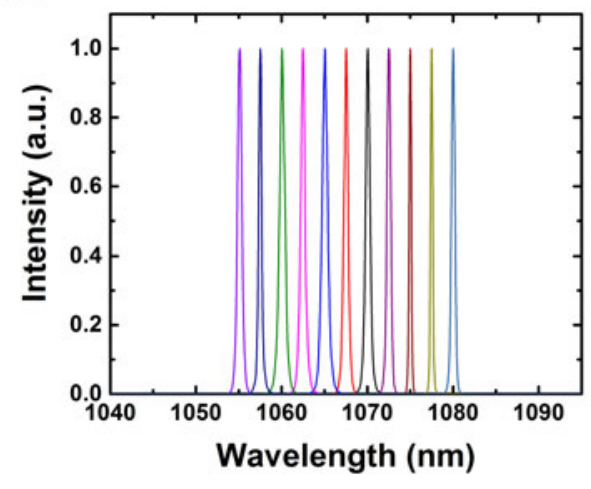

(b)

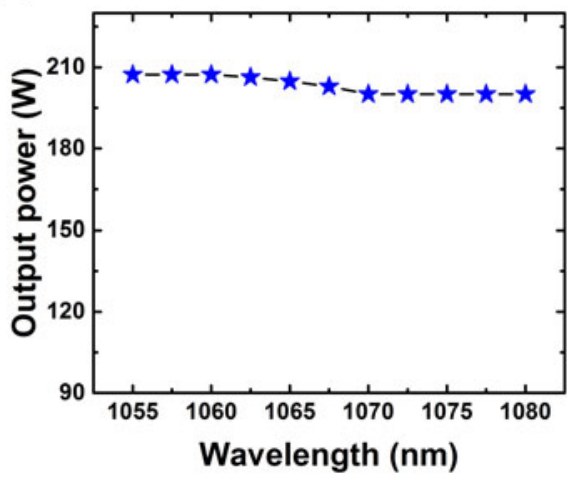

Figure 2. (a) The spectrum and (b) the output power of the tunable pump source.

(a)

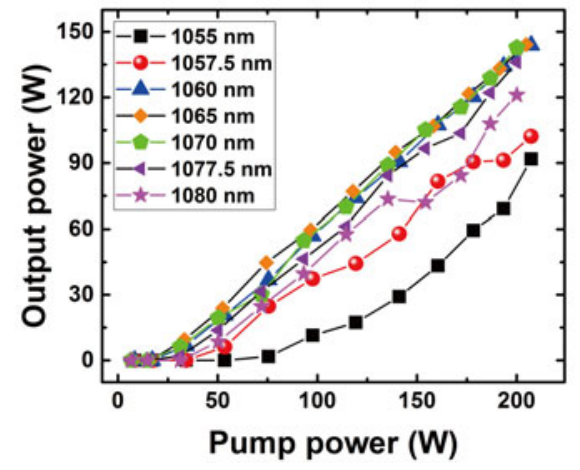

(b)

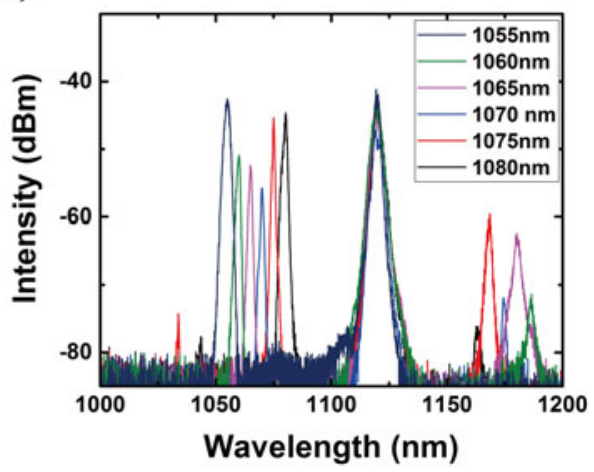

Figure 3. (a) The output power of the first-order Stokes wave versus pump wavelength; (b) the output spectrum as a function of pump wavelength.

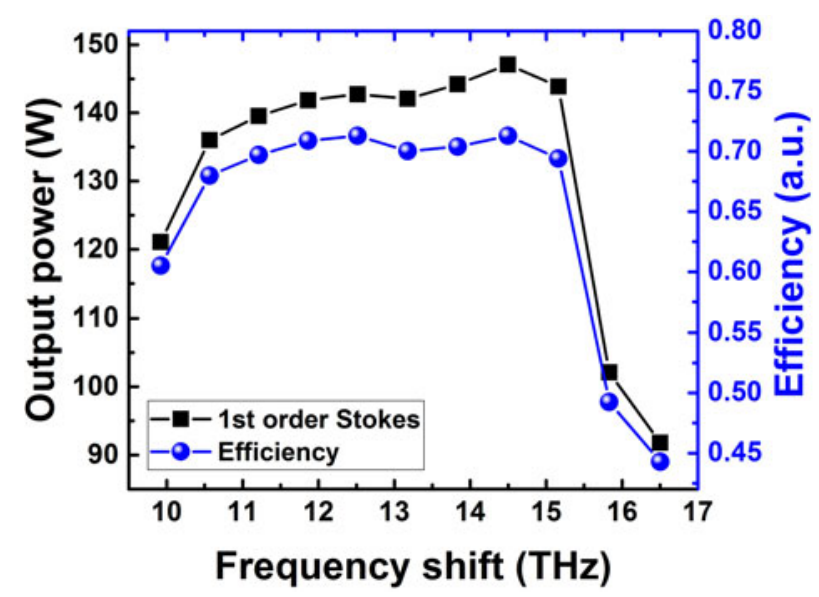

Figure 4. The output power of first-order Stokes wave and corresponding conversion efficiency.

extreme frequency shift between the pump wavelength and Raman wavelength in RFL was studied. The total output power (residual pump plus RFL output power) is around $155 \mathrm{~W}$, corresponding to the slope efficiency of $\sim 77.5 \%$. Figure 3(a) depicts the output power of the first- order Stokes wave at different pump wavelengths. As is shown in Figure 3(b), the frequency shift between the pump wavelength and the first-order Stokes wave and the frequency shift between the first- and second-order Stokes waves are almost the same. This phenomenon may be due to four wave mixing (FWM), which could be further investigated in the future.

The difference among the first-order Stokes wave at different frequency shifts is further explored in Figure 4. As the frequency shift increases, the conversion efficiency of the first-order Stokes increases rapidly when the frequency shift is smaller than $10.6 \mathrm{THz}$, which corresponds to the wavelength longer than $1077.5 \mathrm{~nm}$. At the frequency shift of $14.5 \mathrm{THz}$ corresponding to the pump wavelength of $1062.5 \mathrm{~nm}$, the maximum efficiency of $71.3 \%$ is obtained. From 10.6 to $15.2 \mathrm{THz}$ corresponding to the wavelength from 1077.5 to $1060 \mathrm{~nm}$, the efficiency changes a little around $70 \%$. This efficiency is larger than $95 \%$ of the maximum value, $71.3 \%$. It is to be noted that maximal conversion efficiency locates at the frequency shift of 14.5 THz instead of the theoretical value of $13.2 \mathrm{THz}^{[23]}$. The phenomenon is due to the domination of the second peak in the double-peak structure in Raman gain profile, as was previously modeled based on power balance model 
(a)

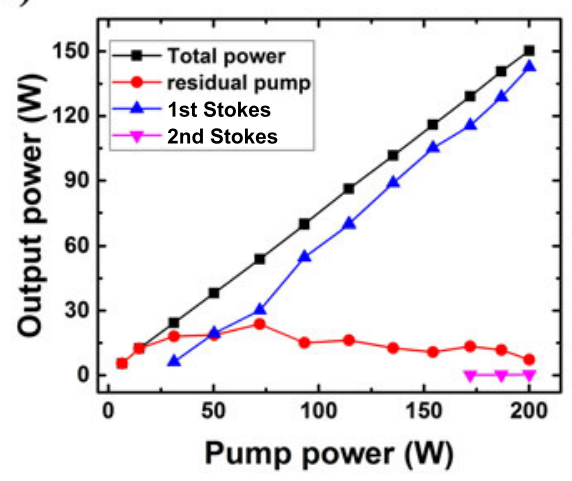

(b)

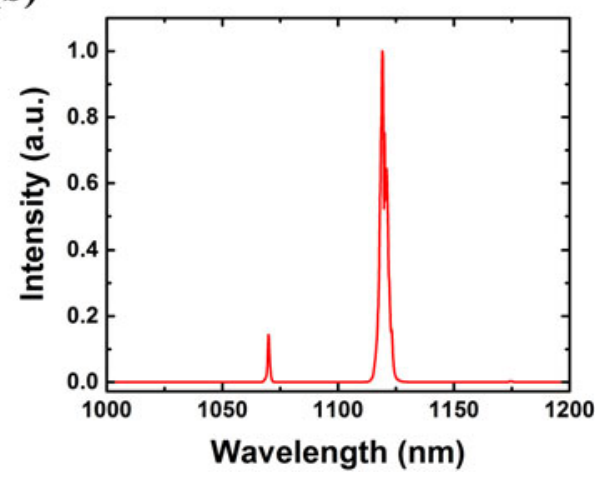

Figure 5. (a) The output power as a function of pump power; (b) the spectrum at maximum power in the linear coordinate.

(a)

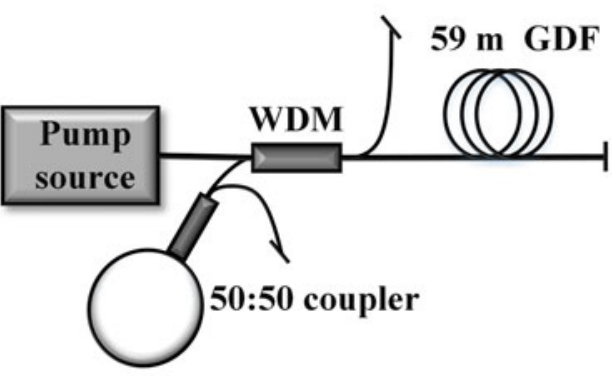

(b)

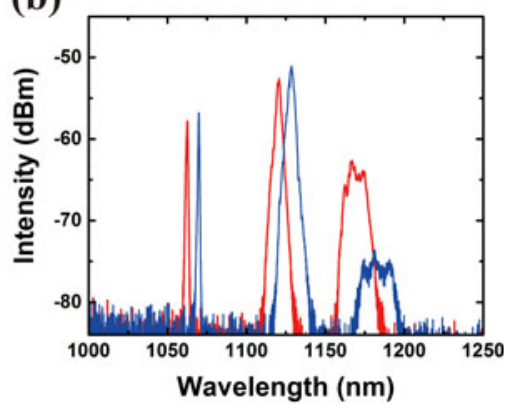

Figure 6. (a) The experimental setup of the comparative experiment; (b) the spectrum of comparative Raman fiber laser at 1062.5 and $1070 \mathrm{~nm}$.

considering different subcomponents with each Stokes component by Vatnik et al. in Ref. [24]. The maximum output power of $147.1 \mathrm{~W}$ is obtained at $1062.5 \mathrm{~nm}$. When the frequency shift is larger than $15.2 \mathrm{THz}$, the efficiency begins to decline. Taking $16.5 \mathrm{THz}$ and $15.2 \mathrm{THz}$ as an example, although the frequency difference between them is only $1.3 \mathrm{THz}$, the difference of efficiency is up to $25.1 \%$. The results indicate that the Stokes frequency downshifted from the pump frequency by 10.6 to $15.2 \mathrm{THz}$ can operate with high power and high efficiency.

The whole system is specially designed to provide almost equal conditions for all the pump wavelengths. Sufficient conversion to the first-order Stokes wave and valid suppression of the second-order Stokes wave should be fulfilled at the same time. Taking the pump wavelength of $1070 \mathrm{~nm}$ as an example, the output properties of the Raman laser are recorded and analyzed. The power evolutions of the total output, residual pump, first-order Stokes wave and second-order Stokes wave are shown in Figure 5(a). The threshold power of Raman lasing is $\sim 30 \mathrm{~W}$. The maximum output power of $1120 \mathrm{~nm}$ Raman laser is $142.7 \mathrm{~W}$ under the pump power of $200.1 \mathrm{~W}$, which corresponds to the optical efficiency of $71.3 \%$. At the maximum pump power, the firstorder Stokes wave occupies $95.0 \%$ of the whole spectrum, while the second-order Stokes wave at $1174.4 \mathrm{~nm}$ merely accounts for $0.2 \%$, as is shown in Figure 5(b). Such a power difference between the first- and second- order Stokes wave is acceptable in our experiment.

Since the stimulated Raman scattering stems from the amplification of spontaneous Raman scattering, we also conducted a comparative experiment in order to further eliminate the effect of the FBGs. As shown in Figure 6(a), the output of the pump source is injected into the $1070 \mathrm{~nm}$ port of a 1070/1120 nm wavelength division multiplexer (WDM). A fiber loop mirror formed by splicing together the two output ports of a 50/50 coupler centering at $1120 \mathrm{~nm}$ and a flat-cut endface are adopted to form a cavity, where the feedback is nearly wavelength independent. A piece of $59 \mathrm{~m} \mathrm{GDF}$, which is longer than that in the Raman oscillator, is used to provide Raman gain in order to decrease the threshold power. The spectra of the output laser at the pump wavelengths of 1062.5 and $1070 \mathrm{~nm}$ are shown in Figure 6(b). The $1062.5 \mathrm{~nm}$ pump laser transforms to $1120 \mathrm{~nm}$ Stokes wave, while $1070 \mathrm{~nm}$ pump source converts to $1128 \mathrm{~nm}$ Stokes wave. The result shows that frequency shift of $\sim 14.5 \mathrm{THz}$ dominates in this laser system. This comparative experiment verifies that the highest conversion efficiency of the $1120 \mathrm{~nm}$ laser at the pump wavelength of $1062.5 \mathrm{~nm}$ is indeed owing to the highest Raman gain.

The PER of the output laser is also measured based on the setup shown in Figure 7. The output laser goes through the collimator to guarantee collimation property. 


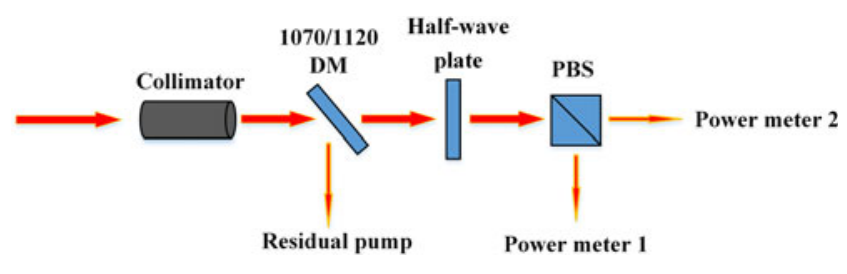

Figure 7. The measurement setup of PER.

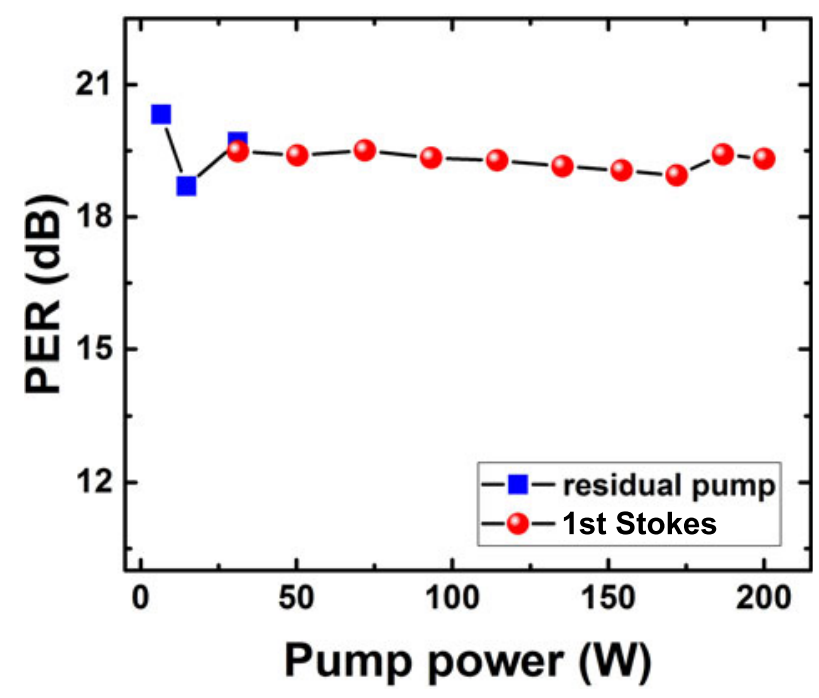

Figure 8. The PER of the residual pump and first-order Stokes wave at the pump wavelength of $1070 \mathrm{~nm}$.

The residual pump and first-order Stokes wave are separated by the $1070 / 1120 \mathrm{~nm}$ dichroic mirror (DM). A half-wave plate working around $1120 \mathrm{~nm}$ is placed after it to adjust the polarization direction. The slow axis and the fast axis are split into two perpendicular directions by a polarization beam splitter (PBS) working around $1120 \mathrm{~nm}$. The output powers of the two beams are recorded by the power meter 1 and power meter 2 , respectively.

The PER of the output laser can be calculated according to PER $=-10 \log (a / b)$, where $a$ and $b$ are the values of minor and major axes of the polarization ellipse, which correspond to the powers measured by the two power meters. The PER of the pump source can be obtained by measuring the PER of the residual pump when the pump power is less than the threshold power of $\sim 30 \mathrm{~W}$, as is shown in Figure 8 . As the pump power increases, the PER evolution of the first-order Stokes wave is measured to be around $19.3 \mathrm{~dB}$ and there is no apparent degradation.

The power stability of the laser system was also measured and recorded, as is shown in Figure 9, the power stability of the output laser under the maximum pump power was monitored and recorded every $10 \mathrm{~s}$ at the time scale of $300 \mathrm{~s}$ by the power meter. The output properties at each pump wavelength were measured every less than $5 \mathrm{~min}$. Therefore, in this case the entire recording time of laser stability is long enough. The standard deviation (STD) normalized to the

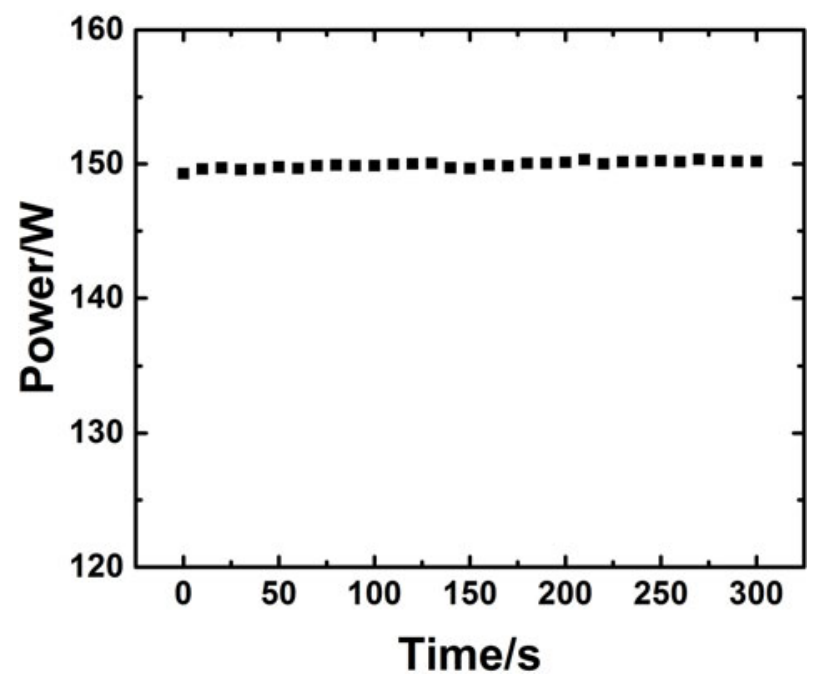

Figure 9. The stability of the output power at the time scale of $300 \mathrm{~s}$.

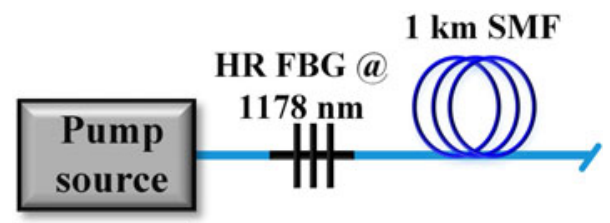

Figure 10. The experimental setup of $1178 \mathrm{~nm}$ Random laser.

mean value of the output power is calculated to be 0.0016 , which indicates that the Raman laser is quite stable.

As indicated in the introduction section, it is interesting to explore the feasibility of generating $1178 \mathrm{~nm}$ Raman laser with $\sim 1110 \mathrm{~nm}$ pump source. The experimental results obtained before have indicated the probability, and now we will validate it through a proof-of-concept experiment. As shown in Figure 10, a $1178 \mathrm{~nm}$ random distributed feedback RFL ${ }^{[34,35]}$ was built based on a half open cavity ${ }^{[36,37]}$. The pump source is a homemade fiber laser centered at $1111.6 \mathrm{~nm}$, which corresponds to the frequency shift of 15.2 THz. An HR FBG and a piece of 1-km-long single mode fiber (SMF) are used to construct the setup of the half open laser cavity. The output endface is angle-cleaved to $8^{\circ}$ to suppress unexpected backward reflection so as to protect the pump source.

The output power and spectra as a function of pump power are shown in Figure 11. As the pump power increases, the output power increases at first and then decreases owing to the generation of second-order Stokes wave. The maximum output power is $11.8 \mathrm{~W}$ under the pump power of $14.7 \mathrm{~W}$. The conversion efficiency of $80.2 \%$ is obtained at the maximum output power, which is sufficient to verify the potential of high power operation at $1178 \mathrm{~nm}$ pumped by high power $1111.6 \mathrm{~nm}$ fiber laser. 
(a)

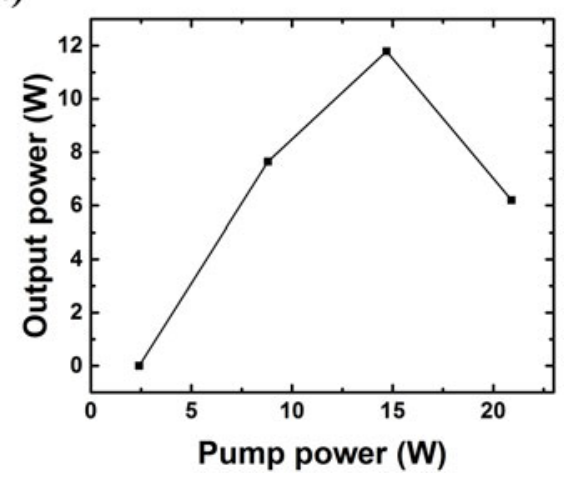

(b)

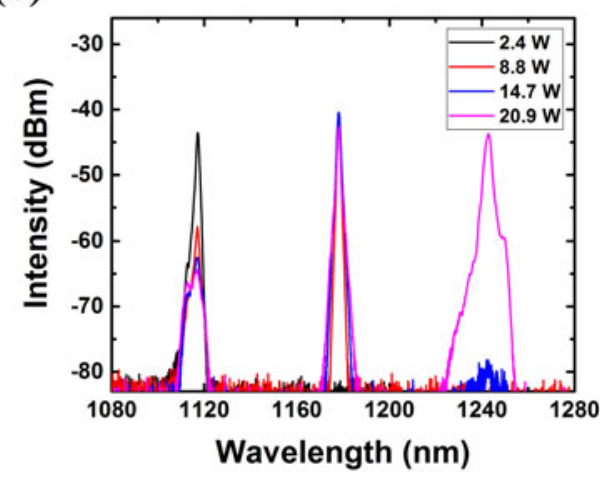

Figure 11. (a) The output power and (b) spectra versus pump power of $1178 \mathrm{~nm}$ random laser.

\section{Conclusion}

In summary, we report an experimental investigation on the extreme frequency shift between the pump wavelength and Raman wavelength in a high-power LP RFL. A pair of FBGs whose central wavelength is $1120 \mathrm{~nm}$ are adopted to achieve fixed target Raman wavelength. A $\sim 200 \mathrm{~W}$ LP wavelength-tunable pump source with $25 \mathrm{~nm}$ tuning range is used to change the frequency shift. A piece of 31-mlong PM silica passive fiber is selected to provide Raman gain. By comparing the properties of power, efficiency and spectra as the pump wavelength tunes from 1055 to $1080 \mathrm{~nm}$, the range of frequency shift that can demonstrate high-power and high-efficiency Raman lasing is achieved. The experimental results verify that Raman laser with the frequency downshifted from the pump frequency by 10.6 to $15.2 \mathrm{THz}$ operates with high efficiency, which is higher than $95 \%$ of the maximum one of $71.3 \%$. At the same time, the maximum output power of $142.7 \mathrm{~W}$ is obtained with an optical efficiency of $71.3 \%$, which is the highest power ever reported in LP RFLs to the best of our knowledge. As a typical application example of the results obtained in this paper, we experimentally verify that the $1120 \mathrm{~nm}$ pump laser could be replaced with a $\sim 1111 \mathrm{~nm}$ pump laser for a $1178 \mathrm{~nm}$ RFL, which is more beneficial to provide high-power pump lasers. The results could help expand the frequency shift in cascaded RFL or select appropriate frequency shift to generate Raman laser at special waveband, while the efficiency would not be reduced compared to the classic frequency shift. The FWM phenomenon observed in the experiment will be further investigated in the near future. The extreme frequency shift in other configurations, such as integrated ytterbium-Raman fiber amplifier and pure Raman amplifiers, could also be investigated in the future.

\section{Acknowledgements}

This work was supported in part by the Huo Yingdong Education Foundation of China (No. 151062), in part by the Foundation for the author of National Excellent Doctoral Dissertation of China (No. 201329), and in part by the National Natural Science Foundation of China (No. 61635005).

\section{References}

1. V. R. Supradeepa, Y. Feng, and J. W. Nicholson, J. Opt. 19, 023001 (2017).

2. V. R. Supradeepa and J. W. Nicholson, Opt. Lett. 38, 2538 (2013).

3. Y. Feng, L. R. Taylor, and D. B. Calia, Opt. Express 17, 23678 (2009).

4. H. Zhang, H. Xiao, P. Zhou, X. Wang, and X. Xu, IEEE Photonics J. 5, 1501706 (2013).

5. Y. Glick, V. Fromzel, J. Zhang, N. Tergabrielyan, and M. Dubinskii, Appl. Opt. 56, B97 (2017).

6. L. Zhang, H. Jiang, S. Cui, and Y. Feng, Opt. Lett. 39, 1933 (2014).

7. H. Zhang, H. Xiao, P. Zhou, X. Wang, and X. Xu, Opt. Express 22, 10248 (2014).

8. L. Zhang, C. Liu, H. Jiang, Y. Qi, B. He, J. Zhou, X. Gu, and Y. Feng, Opt. Express 22, 18483 (2014).

9. Y. Chen, H. Xiao, J. Xu, J. Leng, and P. Zhou, Appl. Opt. 55, 3824 (2016).

10. H. Zhang, R. Tao, P. Zhou, X. Wang, and X. Xu, IEEE Photonics Technol. Lett. 27, 628 (2015).

11. P. Zhou, X. Wang, H. Xiao, Y. Ma, and J. Chen, Laser Phys. 22, 823 (2012).

12. A. S. Kurkov, Laser Phys Lett. 4, 93 (2007).

13. H. Xiao, P. Zhou, X. L. Wang, S. F. Guo, and X. J. Xu, Laser Phys. Lett. 9, 748 (2012).

14. V. Fortin, M. Bernier, D. Faucher, J. Carrier, and R. Vallée, Opt. Express 20, 19412 (2012).

15. X. Jin, X. Du, X. Wang, P. Zhou, H. Zhang, X. Wang, and Z. Liu, Sci. Rep. 6, 30052 (2016).

16. J. W. Nicholson, M. F. Yan, P. Wisk, J. Fleming, F. Dimarcello, E. Monberg, T. Taunay, C. Headley, and D. J. Digiovanni, Opt. Lett. 35, 3069 (2010).

17. H. Zhang, P. Zhou, X. Wang, X. Du, H. Xiao, and X. Xu, Opt. Express 23, 17138 (2015).

18. X. Wang, P. Zhou, H. Zhang, X. Wang, H. Xiao, and Z. Liu, Opt. Lett. 39, 4329 (2014).

19. L. Taylor, Y. Feng, D. Bonaccini Calia, and W. Hackenberg, Proc. SPIE 6272, 627249 (2006).

20. Y. Feng, L. R. Taylor, and D. Bonaccini Calia, Opt. Express 17, 19021 (2009). 
21. A. A. Surin, T. E. Borisenko, and S. V. Larin, Opt. Lett. 41, 2644 (2016).

22. P. T. Rakich, Y. Fink, and M. Soljacić, Opt. Lett. 33, 1690 (2008).

23. G. P. Agrawal, Nonlinear Fiber Optics (Elsevier, 1995).

24. I. D. Vatnik, E. A. Zlobina, S. I. Kablukov, and S. A. Babin, Opt. Express 25, 2703 (2017).

25. H. Zhang, H. Xiao, P. Zhou, K. Zhang, X. Wang, and X. Xu, Appl. Phys. Express 7, 052701 (2014).

26. H. Zhang, H. Xiao, P. Zhou, X. Wang, and X. Xu, IEEE Photonics Technol. Lett. 25, 2093 (2013).

27. J. Wang, J. Hu, L. Zhang, X. Gu, J. Chen, and Y. Feng, Opt. Express 20, 28373 (2012).

28. Y. Jeong, J. K. Sahu, D. N. Payne, and J. Nilsson, Electron. Lett. 40, 470 (2004).

29. Y. Jeong, J. K. Sahu, D. N. Payne, and J. Nilsson, Opt. Express 12, 6088 (2004).
30. R. K. Jain, Chinlon Lin, R. H. Stolen, W. Pleibel, and P. Kaiser, Appl. Phys. Lett. 30, 162 (1977).

31. C. Lin, W. G. French, T. G. Malone, and R. H. Stolen, Opt. Lett. 1, 96 (1977).

32. E. Belanger, M. Bernier, D. Faucher, D. Cote, and R. Vallee, J. Lightwave Technol. 26, 1696 (2008).

33. http://www.ipgphotonics.com/en/products/lasers/mid-powercw-fiber-lasers/1-15-1-8-micron-2/rlm-and-rlr-fiber-lasers-10 0-300.

34. S. K. Turitsyn, S. A. Babin, A. E. El-Taher, P. Harper, D. V. Churkin, S. I. Kablukov, J. D. Ania-Castañón, V. Karalekas, and E. V. Podivilov, Nat. Photonics 4, 231 (2010).

35. X. Du, H. Zhang, H. Xiao, P. Ma, X. Wang, P. Zhou, and Z. Liu, Ann. Phys. Berlin 528, 649 (2016).

36. L. Zhang, H. Jiang, X. Yang, W. Pan, S. Cui, and Y. Feng, Sci. Rep. 7, 42611 (2017).

37. X. Du, H. Zhang, X. Wang, P. Zhou, and Z. Liu, Photon. Res. 3, 28 (2015) 